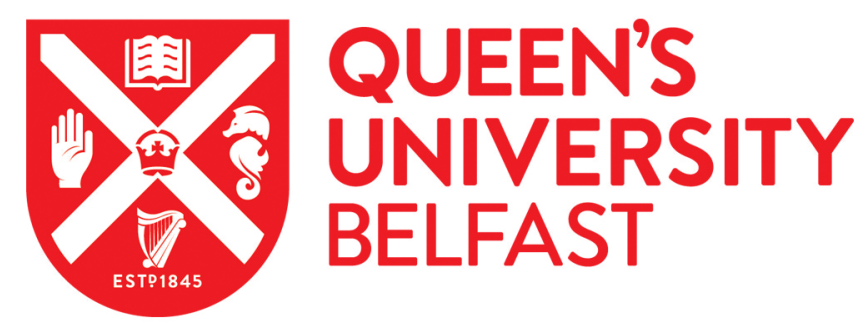

\title{
The relationship between oral-health-related quality of life and general health in an elderly population: a cross sectional study
}

DaMata, C., McKenna, G., Hayes, M., \& Allen, F. (2019). The relationship between oral-health-related quality of life and general health in an elderly population: a cross sectional study. Gerodontology, 36(1), 71-77. https://doi.org/10.1111/ger.12384

\section{Published in:}

Gerodontology

Document Version:

Peer reviewed version

Queen's University Belfast - Research Portal:

Link to publication record in Queen's University Belfast Research Portal

Publisher rights

(C) 2018 Gerodontology Association and John Wiley \& Sons Ltd.

This work is made available online in accordance with the publisher's policies. Please refer to any applicable terms of use of the publisher.

\section{General rights}

Copyright for the publications made accessible via the Queen's University Belfast Research Portal is retained by the author(s) and / or other copyright owners and it is a condition of accessing these publications that users recognise and abide by the legal requirements associated with these rights.

Take down policy

The Research Portal is Queen's institutional repository that provides access to Queen's research output. Every effort has been made to ensure that content in the Research Portal does not infringe any person's rights, or applicable UK laws. If you discover content in the Research Portal that you believe breaches copyright or violates any law, please contact openaccess@qub.ac.uk. 
The relationship between oral-health-related quality of life and general health in an elderly population: a cross sectional study

Running title: Oral health and general health in the elderly

Authors: C da Mata ${ }^{1}$, PF Allen², G McKenna ${ }^{3}$, M Hayes $^{1}$, A Kashan $^{4}$

${ }^{1}$ Cork University Dental School and Hospital, University College Cork, Ireland

${ }^{2}$ Faculty of Dentistry, National University of Singapore

${ }^{3}$ Centre for Public Health, Queens University Belfast, Northern Ireland

${ }^{4}$ Epidemiology \& Public Health, University College Cork, Ireland

\section{Corresponding Author:}

Dr Cristiane da Mata

Restorative Dentistry,

Cork University Dental School and Hospital,

University College Cork,

Ireland

Telephone: (+353) 0214205033 
Fax: (+353) 0214901193

Email: cristiane.damata@ucc.ie

\section{Key Words:}

Oral-health-related quality of life, general health, elderly.

\section{Abstract}

Objective: to assess the oral-health-related quality of life in a cohort of Irish older patients and explore associations with overall health status. Background: The impact of oral health conditions on older individuals' quality of life may be influenced by patients' general health status. Materials and Methods: This paper reports a cross-sectional study, which analysed baseline data from patients aged over 60 years. Patients were recruited from two different environments, namely Cork University Dental Hospital and St. Finbarr's Hospital, to take part in two research studies. All patients completed the Oral Health Impact Profile (OHIP-14) and EuroQol-5D (EQ-5D) questionnaires. All patients provided a comprehensive overview of their general medical status. Data from the Quality of Life questionnaires were analysed to check for differences between healthy and frail elders and explore associations between oral-health-related quality of life and general health. Results: The patient sample comprised 146 (44.6\%) male and 181 (55.4\%) female participants, with a mean (SD) age of 73.96 (6.9). Frail patients reported a higher mean OHIP-14 score compared to non-frail patients ( $p<0.001)$. Pearson's correlation analysis showed a negative association between OHIP-14 and EQ-5D scores. Regression analysis showed that among frail individuals, better general 
health corresponded to poorer oral-health-related quality of life (OHRQoL). In the non-frail cohort, better general health was related to better OHRQoL, although these results were not statistically significant. Conclusions: General health was not significantly associated with the way that patients perceive their oral health within this patient cohort. However, factors such as objective oral health, denture wear and patient's expectations may play a role in this association. 


\section{Introduction}

The impact of population ageing and the shift in the dental status of older adults can already be felt by dentists, policy makers and public health professionals. Individuals are retaining more of their teeth into old age, yet this population still carries a disproportionate burden of oral disease. In many countries, dental caries and periodontal disease are considerable public health challenges amongst the dentate elderly [1]. Oral health provision in this age group is characterised by tooth loss and an ongoing burden of replacement of restorative dental treatment. Furthermore, many older adults experience a significant decline in their oral health as they become more frail, more dependent and cognitively impaired [2]

Oral health is an integral part of the well-being of the individual and essential to general health. In the past, oral health was defined as the absence of chronic oro-facial pain, oral and pharyngeal cancer, oral and dental tissue lesions and other diseases that affect the craniofacial complex. Nowadays, this concept is broader, as the multidimensional nature of health has been recognized. Because oral conditions such as dental caries, periodontitis and missing teeth can result in pain, difficulty with chewing or speech, dissatisfaction with appearance and embarrassment in social contexts, they can have a significant impact on quality of life [3]. Therefore, it would be expected that older patients, who tend to present with poorer oral health, would report worse oral-health-related quality of life (OHRQoL).

However, this often does not seem to be the case, with studies showing that older adults present with better OHRQoL when compared to their younger counterparts. Thus, although tooth loss and other oral conditions appear more frequently in older adults, it seems that some signs and symptoms of oral disease may have a greater impact on subjective oral health when they occur early in adulthood [4]. This is known as the disability paradox. Perhaps the most plausible explanation for this conflicting evidence is that other variables such as social relationships and social context play a part in how people rate their quality of life. Also, other factors may interfere with the processes of coping and adaption [5]. General health issues could be one of these aspects, and previous studies have not investigated the differences in OHRQOL outcomes between groups of patients with different medical status. 
Poor oral and general health are interrelated as they share common risk factors, therefore investigating how older and more frail patients perceive the impact of oral health on their quality of life is very important. The relationship between subjective OHRQoL and general health in elderly patients has been investigated by a very small number of previous studies $[6,7]$. Furthermore, how older adults in poor general health consider the impact of oral health on their overall quality of life compared to fit and healthy elderly is not known.

This study aims to investigate the impact of general health issues on OHrQoL in a cohort of older adults using the short form of the Oral Health Impact Profile (OHIP-14). As the OHIP-14 tool is conceptually based on Locker's model of oral health (Figure 1) it could be assumed that general health problems may affect the way that patients' perceive one or more domains in this model.

Figure 1- Locker's model of oral health

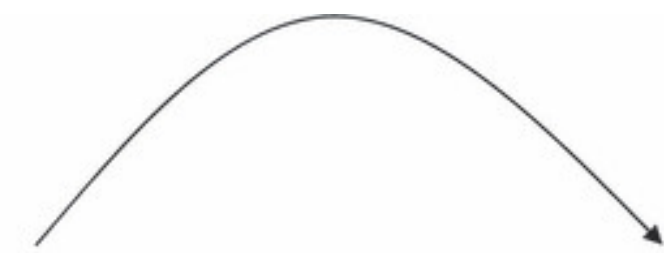

Disease

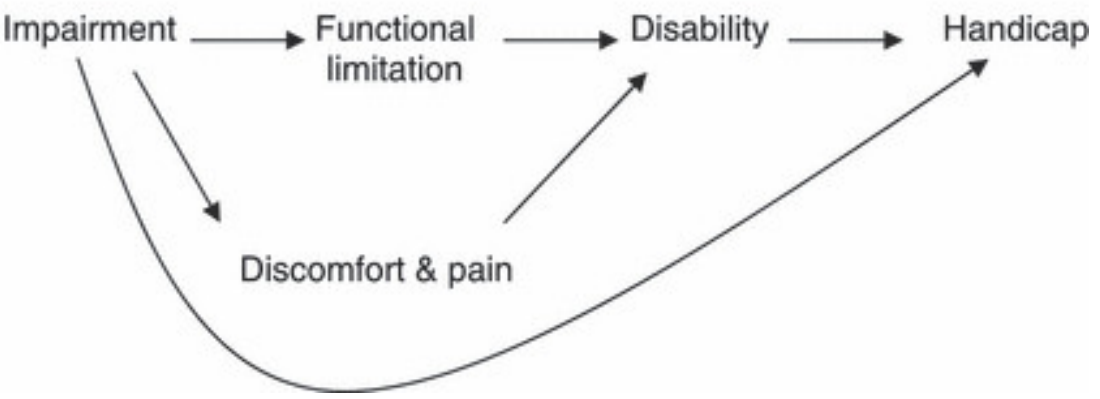

For the purpose of this study we hypothesise no difference in OHRQoL between frail and non-frail elderly (test null hypothesis). Therefore, the objectives of this study are: 
1) To assess the OHRQoL and the self-rated overall health status in a population of independent-living older adults;

2) To assess the association between elderly patients' OHRQoL and overall health status;

3) To compare the OHRQoL and self-rated overall health status of frail and non-frail elderly. 


\section{Methods}

This study aimed to analyse baseline data from two clinical studies which have been undertaken in University College Cork (2008-present). Both studies investigated the impact of different dental treatments on the OHRQoL of older adults. Full ethical approval was granted by the Clinical Research Ethics Committee of the Cork Teaching Hospitals for both studies (ECM 5 (4) 02/09/08, ECM 5 (9) 05/02/08).

\section{Recruitment process}

Although running in parallel, the two studies were carried out separately by two different main investigators. Data were collected from 2 different centres, Cork University Dental School and Hospital (CUDSH) and St Finbarr's Day Hospital (SFH), in Cork.

Patients were recruited by advertisements in the dental school reception area and in a nearby church newsletter. In SFH, patients were approached while in the clinic, and provided with information about the research studies. As a general characterisation, community dwelling older patients were recruited in CUDSH, whilst those in SFH represented an older, more systemically unwell population. None of the patients recruited were from residential care homes.

All patients who agreed to participate were asked to sign a written consent form, and were examined by standardized clinical examiners. Those who met the inclusion criteria to receive treatment were randomly allocated to one of the intervention groups.

The aim of study 1 was to compare two different treatment strategies to restore carious lesions in elderly patients, namely Atraumatic Restorative Treatment and Conventional treatment. Study 2 aimed to replace missing teeth in patients aged over 60 years using either removable partial dentures or resin-bonded bridges. More details of each study, such as inclusion and exclusion criteria, can be found elsewhere $[8,9]$.

Convenience sampling was used in the present study, as patients from two previously conducted studies were included. 


\section{Data collection}

All patients completed a series of baseline questionnaires, which collected demographic data including age, gender, place of residence (e.g., independently living, dependent on home help), medical history, and dental habits. The OHIP-14 [10] and EQ-5D [11] questionnaires were self-completed by patients or by personal interview conducted by the same two trained dentists prior to treatment. Patients then received a clinical examination which included data on decayed, carious and missing teeth (DMFT) . Each intra-oral examination was carried out by two calibrated dentists (inter-examiner consistency kappa score= 0.88 ) who had received prior training by a gold standard examiner.

Instruments used:

1) OHIP-14

The full version of the Oral Health Impact Profile is an oral-health- related quality of life measure developed by Slade \& Spencer (5) and consists of 49 statements organized into seven domains, namely: functional limitation; pain; psychological discomfort; physical disability; psychological disability; social disability and; handicap. The format of the questions is "have you had....because of problems with your teeth, mouth or dentures?", and a Likert response format $(0=$ never, $1=$ hardly ever, $2=$ occasionally, $3=$ fairly often, 4=very often) is used. Summary scores are calculated by summing response codes, and higher scores are indicative of poorer oral health related quality of life. The OHIP-14 is a short version of the OHIP validated by Slade for use in older adults [10] and was chosen for this study because of its shorter length allied with its reliability, validity and precision.

2) EuroQoL 5D (EQ-5D)

The EQ-5D is a worldwide tool for describing health states. It consists of 5 dimensions namely: mobility, self-care, usual activities, pain/discomfort, and anxiety/depression, with 3 possible responses for each dimension to represent no problems (1), some problems (2) and extreme problems (3). Each question will then have the possible answer 
1, 2 or 3 . The five questions will generate a score that could be 12213, meaning answer 1 for question 1, answer 2 for question 2 , answer 2 for question 3 and so on. This summative index represents a certain health state, full health being referred to as 11111 . Weights derived from population-based valuation studies are then used to rate each health state, and scores range from -1 to 1 (with 1 representing full health).

In this present study, weights from the UK valuation of EQ-5D (16) were used as it is the country with most similarities to Ireland.

\section{Defining frailty}

The criteria used to define frailty was based on the Seattle Care Pathway (SCP) Recommendations where patients are categorized as: no dependency, pre-, low, medium and high dependency. These definitions have been linked and share some descriptors of the Canadian Study of Health and Aging Frailty Scores [12].

After examining patients, administering EQ-5D and analyzing their medical history, patients were classified into:

1) no dependency (fit and robust)

2) pre-dependency (well controlled chronic condition, not affecting oral health).

3) low dependency (those with identified chronic conditions affecting their oral health and daily living)

4) medium dependency (those with identified chronic conditions affecting their oral health and daily living, who may need help to access oral services)

5) high dependency ( complex medical condition who cannot be transported to receive dental care)

Patients who fell into category 1 or 2, were classified as non-frail. Patients in category 3 and 4 were considered frail. Patients from SFH fell into these two categories, therefore being categorized as frail. No patients attending these clinics were classified as level 5. 


\section{Data analysis}

Data were stored in two different files, in IBM SPSS Statistics for Windows, Version 22.0. Armonk, NY: IBM Corp., which were checked for accuracy and merged.

The variables were summarized using absolute numbers with percentages, mean, median, and confidence intervals.

Given the large sample size, and only slight deviations from normality, Students' t-test was used to test the hypothesis that the distribution of OHIP scores was equal across different groups. The continuous variable OHIP score was correlated with EQ-5D score using Pearson's correlation coefficients. 95\% Cls were calculated using Fisher's z transformation.

Linear regression analysis was used to measure the association between the dependent variable oral-health-related quality of life (OHIP) and self-rated general health (EQ-

5D). The assumptions of independence, homoscedasticity and normality of standardized residuals were checked. The models were adjusted for variables whose outcomes have been found to influence OHRQoL (i.e. gender, age, frailty status).

Dental data was summarized using percentages, means and confidence intervals. Students' t-test was used to compare mean number of DMFT, decayed and missing teeth between the frail and non-frail groups.

\section{Results}

Demographics and summary statistics

In total, data were collected for 331 patients, 199 patients from study 1 and 132 from study 2 . Three individuals were excluded from the final analysis due to missing OHIP-

14 data. No subjects recorded missing responses for the EQ-5D. Therefore, data for 327 patients were analysed. Overall, the patient sample comprised 146 (44.6\%) male 
and 181 (55.4\%) female participants, with a mean age of 73.96 (Min: 60.58, max: 91.53, SD: 6.9). Nearly a half of all patients (43.4\%- 142/327) were aged between 70 and $80,36.3 \%$ between 60 and 70 and $20.2 \%$ were over 81 years of age.

All participants were independently living elderly. Sixty-four per cent of the patients (209/327) stated they were receiving some medical treatment at the time of data collection and $90.8 \%$ (297/327) reported that they were regularly taking prescribed medication. Table 1 shows demographic profile of all participants. The mean OHIP-14 score recorded for the entire patient sample was 9.3 (SD: 7.4). The scores increased (worsening OHRQoL) as age increased. When frail and non-frail elderly were compared, frail patients presented higher (worse quality of life) OHIP-14 scores (mean=12.8, SD=6.3) compared to non-frail individuals (mean=7.9, SD=7.3). This difference was statistically significant $(p<0.001)$. The mean (SD) EQ-5D score of the entire sample was $0.79(0.23)$. Frail and non-frail patients presented a mean (SD) EQ-5D score of $0.66(0.27)$, and $0.84(0.19)$, respectively.

Table 1- Participants' profile

\begin{tabular}{|c|c|c|c|}
\hline & Overall (N=327) & Frail $(\mathrm{N}=93)$ & Non-frail $(N=234)$ \\
\hline Mean Age (SD) & 73.9 yrs (6.9) & 79.2 & 71.8 \\
\hline $\begin{array}{c}\text { Gender } \\
\text { Males } \\
\text { Females }\end{array}$ & $\begin{array}{l}\mathbf{N}(\%) \\
146(44.6 \%) \\
181(55.4 \%)\end{array}$ & $\begin{array}{l}47.3 \%(44) \\
52.7 \%(49)\end{array}$ & $\begin{array}{l}43.6 \%(102) \\
56.4 \%(132)\end{array}$ \\
\hline On medication & 297 (91.7\%) & $92(98.8 \%)$ & $2017(88.7 \%)$ \\
\hline Currently being treated by a doctor & $211(65.1 \%)$ & $90(96.5 \%)$ & $121(51.6 \%)$ \\
\hline Smokers & $40(12.3 \%)$ & $18(20.9 \%)$ & $22(9 \%)$ \\
\hline OHIP-14 & $\begin{array}{l}\text { Mean (SD) } \\
9.3(7.4)\end{array}$ & 12.8 (SD 6.3) & 7.9 (SD 7.3) \\
\hline$E Q-5 D$ & $0.79(0.73)$ & 0.65 (SD 0.27) & 0.84 (SD 0.19) \\
\hline DMFT & $25.6(4.8)$ & $28(4.3)$ & $24(4.7)$ \\
\hline
\end{tabular}




\begin{tabular}{l|l|l|l} 
Decayed teeth & $2.3(2.7)$ & $3.8(2.7)$ & $1.7(2.5$ \\
\hline Missing teeth & $14.8(5.9)$ & $16.6(5.8)$ & $14(5.8)$ \\
\end{tabular}

Figure 2- Relationship between OHIP scores and EQ-5D 


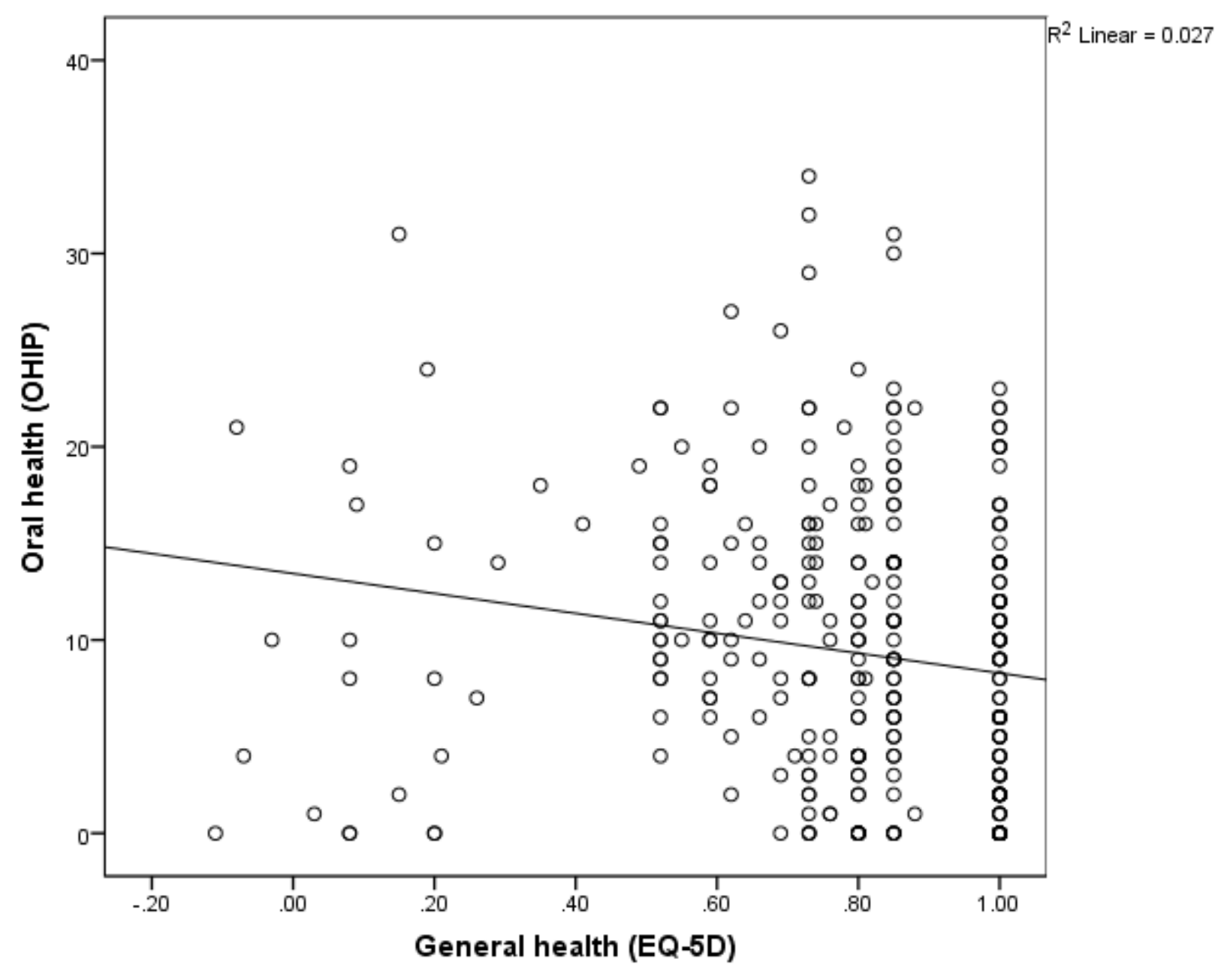


In the linear regression analysis, the crude model showed a significant decrease in OHIP-14 scores (-5.1; [95\% CI: $-8.5,-1.7])$ with one unit increase in EQ-5D. The adjusted model showed a smaller and non-significant difference $(-2.3[95 \% \mathrm{Cl}:-5.8,1.1])$. The main confounder seemed to be recruitment centre (frail and non-frail patients) so, the analysis was repeated for each centre. Frail patients' OHIP-14 scores increased 3.6 units with every unit increase in EQ-5D [95\% CI: -1.0, 8.2]), whereas among non-frail patients, OHIP scores decreased 6.0 units $[95 \% \mathrm{Cl}:-10.7,-1.3])$ with every unit increase in EQ-5D. The adjusted model was a better predictor of variances in OHRQoL in non-frail $\left(R^{2}=0.16\right)$ compared to frail individuals $\left(R^{2}=0.06\right)$. Table 2 shows the results of the regression model (crude, adjusted and adjusted according to frailty status).

Table 2- Multiple linear regression assessing correlation between OHIP scores and EQ-5D

\begin{tabular}{|c|c|c|c|c|c|}
\hline & $\begin{array}{l}\text { Mean } \\
\text { Difference }\end{array}$ & Standardized $\beta$ & P-value & $95 \% \mathrm{CI}$ & Adjusted R2 \\
\hline \multicolumn{6}{|l|}{ Crude model } \\
\hline EQ-5D & -5.15 & -0.16 & 0.003 & $-8.53,-1.77$ & 0.02 \\
\hline \multicolumn{6}{|l|}{ Adjusted model } \\
\hline EQ-5D & -2.31 & -.074 & 0.19 & $-5.82,1.19$ & 0.12 \\
\hline Gender & 0.49 & 0.03 & 0.52 & $-1.04,2.03$ & \\
\hline Age & -0.02 & -0.01 & 0.75 & $-0.14,0.10$ & \\
\hline Smoking status & -1.07 & -0.04 & 0.37 & $-3.43,1.29$ & \\
\hline Frailty status & -3.77 & -0.23 & 0.000 & $-5.80,-1.73$ & \\
\hline \multicolumn{6}{|l|}{ Frail group } \\
\hline EQ-5D & 3.62 & 0.15 & 0.12 & $-1.01,8.27$ & 0.06 \\
\hline \multicolumn{6}{|l|}{ Non-frail group } \\
\hline EQ-5D & -6.0 & -0.15 & 0.13 & $-10.7,-1.3$ & 0.16 \\
\hline
\end{tabular}

Frail vs non-frail individuals 
From the total sample, 93 patients came from SFH and 234 from CUDSH. SFH patients comprised an older cohort, with a mean (SD) age of 79.21 (6.85) compared to 71.79 (5.84) in CUDSH. The majority of patients in SF were females (52.7\%) as it was the case in the CUDSH (56.4\%). The EQ-5D mean (SD) score was 0.66 (0.27) for SFH patients compared to $0.84(0.19)$ in CUDSH patients. A greater number of SFH patients (62.4\%) reported mobility problems, compared to CUDSH patients (17.9\%). Also, $44.1 \%$ of SFH patients presented self-care problems. When OHIP scores were compared, frail patients presented higher mean scores (12.8) compared to non-frail individuals (7.9) $(p<0.001)$. Frail individuals presented a mean DMFT of 28 , compared to 24 in non-frail elderly $(p<0.001)$, with higher numbers of decayed and missing teeth (Table 1 ). 


\section{Discussion}

This study found a small degree of negative correlation between OHRQoL and self-perceived general health. As OHIP-14 scores decreased (improved OHRQoL), EQ-5D increased (improved self-rated general health). Therefore, despite the weak correlation, this relationship was in the expected direction. The multiple regression results showed then that when controlling for gender, age, smoking status, frailty status and study which patients had come from, self-rated general health wasn't a significant predictor of OHRQoL. A small number of other studies have assessed the relationship between oral and general health but the findings are not conclusive, with some of them finding no association between the two entities [7], whereas others found that self-rated general health and oral-health-related quality of life are correlated [13].

In this study, frailty status appeared to be the main confounder and the analysis was then repeated for each group (frail and non-frail) separately. Although there was still no significant association between OHRQoL and self-rated general health, an interesting finding was the different direction of change in OHIP with better general health, when frail and non-frail were compared. Non-frail individuals with better subjective general health presented better OHRQoL, which would be expected as oral health is part of general health. However, among frail individuals OHIP scores were worse with better general health. The poorer oral health status among frail compared to nonfrail individuals as shown by DMFT, number of decayed and number of missing teeth, could help explain the different direction of change in OHIP with better general health in this group, as better health might change patients' focus to dental problems, which would reflect in poorer oral-health-related quality of life. Patients' expectations of dental treatment, oral health attitudes, and personality traits [14] might also be different in frail compared to non-frail individuals, and could influence the relationship between OHIP and EQ-5D scores. Other factors that could mitigate this relationship could be an individual' overall quality of life and happiness levels. Locker et al. (2000) found a significant association between self-perceived oral health and morale, life satisfaction and perceived life stress. Although this could show the impact of OHRQoL, it would also be plausible to consider that psychological problems such as low morale or stress lead people to perceive their oral health more negatively [15], which might be the case with those undergoing multiple medical treatments. General health and subjective oral health have also been recently shown to be associated 
with happiness levels in the elderly in a Korean study [16]. The difference between frail and non-frail patients found here could also be the result of a lack of statistical power, given the smaller number of frail patients.

The overall OHIP-14 score in this population of independent-living Irish elderly was 9.3 (SD:7.4). The scores were higher (worse OHRQoL) with increased age. Previous studies have reported lower OHIP-14 scores for older individuals (over 65 years) when compared to the scores found here and have also demonstrated a pronounced inverse age-association with OHIP-14 scores $[4,17]$. We found that OHIP-14 scores increased with age, showing that the oldest old were more bothered by their oral health compared to the youngest old patients. This would contradict the disability paradox, which states that oral disease might have less of an impact when it happens in older age. However, we must be careful when comparing our results to those previously mentioned studies, as besides being cross-sectional national dental surveys, they all compared older individuals (over 65 ) to younger cohorts. In this trial, all patients were over 60 and therefore we were comparing younger old to older old individuals.

Patients in SFH presented with lower EQ-5D scores, which is line with our classification of these patients as frail. It had been hypothesised that this cohort would present lower OHIP scores (better subjective oral health), as they were more systemically unwell and older compared to patients from CUDSH. Nonetheless, SFH patients presented with higher OHIP scores, meaning that even being older and in poorer health than CUDSH patients, their oral health status still impacted on their quality of life. One could argue that the hospital setting might have an impact on patients' well- being and consequently on their quality of life. However, although frail and non-frail patients were recruited from different settings in this study, none of them were in-patient environments, and patients attended as out-patients only.

Therefore, the main finding of the present study was that the group of older patients can be very heterogeneous, and classifying all people over 60 years of age as being elderly is a very simplistic approach. When conducting studies involving older adults, accounting for the differences between the old, and the very old is important as well as between frail and non-frail individuals. Taking these differences into account could design more appropriate treatment interventions to each one of them. 
We used subjective measures of oral health and general health, which are nowadays considered valid outcome measures for understanding patients' views and preferences. This will lead to more patient-centred policies and are important considerations for medical-decision making [18]. Although it is known that impaired dental status is associated with deteriorations in oral health-related quality of life, the use of other oral health status measures such as BPE, presence and status of dentures and presence of dental pain could have helped understand the differences in OHRQoL encountered in the present study.

Also, in regards to oral health and its association with general health, multi-centred trials with collection of subjective and objective data might neutralize the heterogeneity between the two recruitment centres used here and contribute to better understanding the relationship between oral and general health.

\section{Conclusion}

Frail elderly presented with poorer OHRQoL compared to healthier individuals, and although OHRQoL and general health might be associated in elderly patients, only a small negative association was found in the present study. OHIP-14 showed a different pattern of association with general health when frail and non-frail elderly were compared, and the use of larger sample sizes with detailed oral health data combined with subjective measures might beneficial in explaining these differences. 


\section{References}

1. Petersen, P., et al., Global oral health of older people-call for public health action. Community dental health, 2010. 27(4): p. 257-67.

2. Chalmers, J.M., Minimal intervention dentistry: part 1. Strategies for addressing the new caries challenge in older patients. Journal of the Canadian Dental Association, 2006. 72(5).

3. Locker, D., Measuring oral health: a conceptual framework. Community Dent Health, 1988. 5: p. 3-18.

4. Slade, G.D. and A.E. Sanders, The paradox of better subjective oral health in older age. Journal of dental research, 2011. 90(11): p. 1279-1285.

5. Albrecht, G.L. and P.J. Devlieger, The disability paradox: high quality of life against all odds. Social science \& medicine, 1999. 48(8): p. 977-988.

6. Zenthöfer, A., et al., Determinants of oral health-related quality of life of the institutionalized elderly. Psychogeriatrics, 2014. 14(4): p. 247-254.

7. Niesten, D., et al., Oral health-related quality of life and associated factors in a care-dependent and a careindependent older population. Journal of dentistry, 2016. 55: p. 33-39.

8. da Mata, C., et al., Two-year survival of ART restorations placed in elderly patients: A randomised controlled clinical trial. Journal of dentistry, 2015. 43(4): p. 405-411.

9. McKenna, G., et al., The impact of rehabilitation using removable partial dentures and functionally orientated treatment on oral health-related quality of life: a randomised controlled clinical trial. Journal of dentistry, 2015. 43(1): p. 66-71.

10. Slade, G.D., Derivation and validation of a short-form oral health impact profile. Community dentistry and oral epidemiology, 1997. 25(4): p. 284-290.

11. Rabin, R. and F.d. Charro, EQ-SD: a measure of health status from the EuroQol Group. Annals of medicine, 2001. 33(5): p. 337-343.

12. Pretty, I.A., et al., The Seattle Care Pathway for securing oral health in older patients. Gerodontology, 2014. 31(s1): p. 77-87.

13. Zimmer, S., et al., Association between oral health-related and general health-related quality of life in subjects attending dental offices in Germany. Journal of public health dentistry, 2010. 70(2): p. 167-170.

14. Takeshita, H., et al., Association of personality traits with oral health-related quality of life independently of objective oral health status: a study of community-dwelling elderly Japanese. Journal of dentistry, 2015. 43(3): p. 342-349.

15. Locker, D., M. Clarke, and B. Payne, Self-perceived oral health status, psychological well-being, and life satisfaction in an older adult population. Journal of dental research, 2000. 79(4): p. 970-975.

16. Yoon, H.S., et al., Happiness, subjective and objective oral health status, and oral health behaviors among Korean elders. Community dentistry and oral epidemiology, 2013. 41(5): p. 459-465.

17. Kotzer, R.D., et al., Oral health-related quality of life in an aging Canadian population. Health and quality of life outcomes, 2012. 10(1): p. 50.

18. Gil-Montoya, J.A., et al., Oral health in the elderly patient and its impact on general well-being: a nonsystematic review. Clinical interventions in aging, 2015. 10: p. 461. 